\title{
Espacio de interacción y trabajo colaborativo en un taller matemático: Winplot en un estudio sobre sólidos de revolución
}

\author{
Sonia Maria da Silva Junqueira \\ soniajunqueira@unipampa.edu.br \\ https://orcid.org/0000-0002-5616-5344 \\ Leandro Blass \\ leandroblass@unipampa.edu.br \\ https://orcid.org/0000-0003-2302-776X \\ Lucas Capello \\ lucascapello@hotmail.com \\ https://orcid.org/0000-0003-0076-5516
}

Universidade Federal do Pampa (UNIPAMPA, Brasil)

Recibido: 30/05/2020 Aceptado: 06/07/2020

\begin{abstract}
Resumen
En vista de las dificultades expuestas por la mayoría de los estudiantes de Educación Superior relacionadas con la comprensión del contenido de sólidos de revolución de la disciplina de Cálculo II, este trabajo propone mostrar resultados sobre algunas actividades dirigidas a este estudio con la ayuda del software Winplot. Por lo tanto, este artículo tiene como objetivo presentar elementos de interacción, observados en una propuesta de aprendizaje colaborativo, desde la aplicación de un taller a estudiantes de pregrado en UNIPAMPA y buscó responder cómo se muestra el proceso de interacción entre los sujetos y entre los sujetos y los objetos a conocer, considerando el apoyo educativo de Winplot. El enfoque metodológico fue cualitativo, en una investigación exploratoria y análisis argumentativo de los datos. Según el estudio, se descubrió que la interacción colaborativa se destaca em elementos de simetría de acción, conocimiento y estado. Aun así, Winplot amplió el campo de representación y visualización para la realización de conjeturas e hipótesis, contribuyendo a fomentar los mecanismos de negociación necesarios para el aprendizaje colaborativo.
\end{abstract}

Palabras clave: Aprendizaje colaborativo; Winplot; Contextos de aprendizaje activo y colaborativo; Pensamiento Matemático.

\section{Espaço de interação e trabalho colaborativo em uma oficina matemática: $O$ Winplot em um estudo sobre sólidos de revolução}

\section{Resumo}

Diante das dificuldades expostas por grande parte dos estudantes do Ensino Superior relacionadas à compreensão do conteúdo de sólidos de revolução da disciplina de Cálculo II, propõem-se neste trabalho mostrar resultados sobre atividades voltadas para esse estudo com o auxílio do software Winplot. Assim, este artigo tem como objetivo apresentar elementos da interação, observados em uma proposta de aprendizagem colaborativa, a partir da aplicação de uma oficina a estudantes de cursos de graduação da UNIPAMPA buscou responder como se 
mostra o processo de interação entre os sujeitos e entre os sujeitos e os objetos a conhecer, considerando-se o apoio pedagógico do Winplot. A abordagem metodológica foi qualitativa, em uma pesquisa exploratória e análise argumentativa dos dados. De acordo com o estudo, constatou-se que a interação colaborativa é destacada em elementos de simetria de ação, de conhecimento e de status. Ainda, o Winplot ampliou o campo de representação e visualização para a realização de conjecturas e hipóteses, contribuindo para fomentar mecanismos de negociação necessários à aprendizagem colaborativa.

Palavras-chave: Aprendizagem colaborativa; Winplot; Contextos de aprendizagens ativas e colaborativas; Pensamento Matemático.

\title{
Space for interaction and collaborative work in a mathematical workshop: Winplot in a study on revolution solids
}

\begin{abstract}
In view of the difficulties exposed by most students of Higher Education related to the understanding of the content of Revolution Solids of the discipline of Calculus II, this work proposes show results about some activities aimed at this study with the help of the Winplot software. Thus, this article aims to present elements of interaction, observed in a collaborative learning proposal, from the application of a workshop to undergraduate students at UNIPAMPA and sought to answer how the interaction process between subjects and between the subjects and objects to know, considering the educational support of Winplot. The methodological approach was qualitative, in an exploratory research and argumentative analysis of the data. According to the study it was found that the collaborative interaction ie highlighted in elements of action symmetry, knowledge and status. Still, Winplot expanded the field of representation and visualization for the realization of conjectures and hypotheses, contributing to foster the necessary negotiation mechanisms for collaborative learning.
\end{abstract}

Keywords: Collaborative learning; Winplot; Contexts of active and collaborative learning; Mathematical Thinking.

\section{Introdução}

Esta pesquisa se insere no âmbito do Grupo de Pesquisa sobre Aprendizagens, Metodologias e Avaliação ${ }^{1}$ (GAMA) e projeto de Pesquisa Laboratório de Investigações Matemáticas Ativas"2, e tem como objetivo apresentar elementos da interação, observados em uma proposta de aprendizagem colaborativa, a partir da aplicação de uma oficina com estudantes dos cursos de Matemática e Engenharia da Universidade Federal do Pampa, UNIPAMPA/Campus Bagé/RS.

\footnotetext{
${ }^{1}$ Grupo de pesquisa registrado no diretório de grupos no CNPq.

${ }^{2}$ Projeto de pesquisa cadastrado no SIPPEE/UNIPAMPA, código de registro 20170808161710.
} 
Como problemática de pesquisa, destaca-se o contexto dos desafios para o ensino e aprendizagem da matemática refletidos nos baixos índices de desempenho de estudantes brasileiros. De acordo com a pesquisadora Sadovsky (2007), o baixo desempenho dos estudantes em matemática é um problema de âmbito global, sendo reflexo de um ensino que se remete quase exclusivamente a decorar fórmulas e procedimentos mecanizados, sobre os quais são raros os que compreendem quando e como utilizá-los. A existência de um ensino memorístico, mecânico e superficial é retratado por Moraes (2008), que expressa a necessidade de uma melhor articulação entre os conteúdos e os procedimentos metodológicos, para assim permitir ao aluno a apropriação dos conhecimentos da matemática de forma significativa.

A fim de enfrentar essa problemática, metodologias alternativas têm sido desenvolvidas e aplicadas por professores de matemática em diferentes níveis do ensino, a maioria delas orientadas para as aprendizagens ativas (Bonwell \& Eison, 1991; Felder \& Brent, 2009; Vickery, 2016; Weltman, 2007).

Nessa direção, a utilização de softwares, de acordo com Pacheco e Barros (2013), pode tornar-se uma proposta dinâmica de apoio pedagógico, capaz de romper com a postura passiva dos estudantes, além de motivar para as aprendizagens ativas, quando submetida a um planejamento estruturado para essa finalidade.

Um desses softwares é o Winplot ${ }^{3}$, que permite a criação, edição e visualização em 2D (duas dimensões) e 3D (três dimensões) em modo compatível ao estudo de diferentes tipos de equações (explícitas, implícitas, paramétricas), além de proporcionar animação gráfica. Dentre as funcionalidades matemáticas da ferramenta é possível estimar ou gerar aleatoriamente uma equação, assim como permitir ajustes posteriores ao gráfico gerado, dentre os quais se incluem configurações em tempo real de seleção, de coordenadas, de tabelas e escalas de eixos. O software é gratuito e apresenta alto grau de interatividade e funcionalidade matemática.

Em vista desses aspectos, o trabalho colaborativo foi idealizado para comportar a utilização do software Winplot por meio da aplicação da oficina denominada: "Utilização do Winplot para o estudo de sólidos de revolução". Como público alvo contou com estudantes que haviam concluído ou estavam cursando o componente curricular de Cálculo II na

\footnotetext{
${ }^{3}$ Winplot - download em https://winplot.br.jaleco.com/.
} 
UNIPAMPA/Campus Bagé. O Winplot foi usado como um apoio pedagógico aos participantes, para a plotagem, rotação, visualização de gráficos de funções específicas para a oficina e subsídio para confronto em relação aos cálculos dos volumes gerados nas atividades propostas.

Desse modo, este estudo priorizou o trabalho colaborativo com o objetivo de levantar elementos da interação entre os sujeitos e entre os sujeitos e objetos a conhecer, observados durante a aplicação da oficina. Considera-se que a aprendizagem colaborativa, conforme propõe Dillenbourg (1999), possa assumir diferentes características. Portanto, os resultados alcançados são variáveis, a depender de diversos fatores, como o grau de simetria de interação entre os sujeitos em uma nova aplicação da mesma oficina. Neste estudo, destaca-se que os elementos da interação estão revelados essencialmente na simetria de ação, de conhecimento e de status entre os participantes, demonstrados no trabalhar juntos com um objetivo compartilhado.

\section{Discussão teórica}

Na sequência são apresentadas reflexões teóricas e introdutórias, primeiramente acerca da interação em processos voltados à aprendizagem colaborativa. No segundo momento, sobre a interação possibilitada pelo software Winplot no âmbito dos pensamentos matemáticos e geométricos.

\section{Aprendizagem colaborativa: reflexões introdutórias}

A aprendizagem colaborativa não é um conceito atual, tem sido aplicada desde o século XVIII por seus defensores, por a considerarem, juntamente com a aprendizagem cooperativa e trabalho em grupo, como iniciativas que contribuem para potencializar e preparar os estudantes para os enfrentamentos do mundo real. Nessa direção, formas colaborativas de ensinar e aprender podem tornar os alunos mais responsáveis por sua aprendizagem, uma vez que podem desencadear mecanismos de aprendizagem como a assimilação de conceitos e a construção autônoma de conhecimentos (Torres \& Irala, 2014).

De acordo com Dillenbourg (1999), a aprendizagem colaborativa não é um mecanismo e nem um método. Há que se tomar cuidado em não cair em uso obsessivo do termo. Para esse 
autor não faz sentido falar efeitos cognitivos de situações colaborativas se qualquer situação for rotulada como colaborativa. Assim, aponta que não é um mecanismo, pois sistemas cognitivos individuais não aprendem porque são individuais, mas porque executam algumas atividades (leitura, construção, predição, etc.) que acionam mecanismos de aprendizagem (indução, dedução, compilação, etc.). Da mesma forma, pares não aprendem porque são dois, mas porque executam algumas atividades que desencadeiam mecanismos de aprendizagem específicas. Isso inclui as atividades/mecanismos realizados individualmente, uma vez que a cognição do indivíduo não é suprimida na interação entre pares. Mas, além disso, a interação entre os indivíduos gera atividades extras (explicação, a discordância, a regulação mútua, etc.), que desencadeiam mecanismos cognitivos extras (elicitação de conhecimento, internalização, redução de carga cognitiva, etc.).

Nessa direção, aprender com colaboração significa também aprender individualmente, pois um fato não elimina o outro. Indivíduos aprendem porque realizam tarefas como leitura, construção, previsão, etc., que acionam alguns mecanismos de aprendizagem. De modo correlato, grupos não aprendem porque são mais do que um, ou porque duas cabeças pensam melhor do que uma. De fato, ocorre que realizam atividades adicionais, que geram os mecanismos cognitivos extras que favorecem a obtenção do conhecimento, a internalização, etc. Para Dillenbourg (1999), a atividade individual ou os mecanismos cognitivos realizados individualmente não estão suprimidos na interação em grupos. A aprendizagem colaborativa considera essas atividades e também os mecanismos extras, que podem ocorrer com mais frequência em situações colaborativas do que na condição exclusiva do trabalho individual.

Aprendizagem colaborativa não é um método em razão da baixa previsibilidade dos tipos específicos de interações. Basicamente, a aprendizagem colaborativa assume formas distintas para os sujeitos (Dillenbourg, 1999), por exemplo, em forma de instruções -vocês têm que trabalhar juntos; referindo-se a um ambiente compartilhado -companheiros de equipe trabalham na mesma mesa e a restrições institucionais- cada membro do grupo receberá a nota atribuída ao projeto do grupo. Portanto, uma situação colaborativa é uma espécie de contrato social (Dillenbourg, 1999), seja entre os pares ou entre os pares e o professor, como um contrato didático (Brousseau, 1986, 1996). Este contrato especifica condições sob as quais alguns tipos de interações. 
Assim, colaborar significa trabalhar junto, em um processo interativo, tendo em vista um objetivo comum, otimizando interações que viabilizem a aprendizagem. A definição mais ampla de aprendizagem colaborativa para Dillenbourg (1999) considera uma situação em que “duas ou mais” pessoas “aprendem” ou tentam aprender algo “juntas”. Há uma relação de escala nessa estrutura, pois há que se considerar o número de sujeitos da colaboração (duas ou mais pessoas: 2,3 a 5, 10, 100, 1000, ...); a que se refere a aprendizagem (aprender algo: é seguir o curso, executar as atividades propostas, aprender ao longo da vida, ...) e as diferentes formas de interação (juntos: presencial ou por computador, síncrona ou assíncrona, frequente no tempo ou não, esforço conjunto ou dividido, ...).

Ainda, uma situação é colaborativa se os pares se encontram mais ou menos no mesmo nível, são capazes de realizar as mesmas ações, tem um objetivo comum e trabalham sincronamente. Isso se explica nos termos utilizados por Dillenbourg (1999) que situações caracterizadas como colaborativas apresentam i) simetria na interação: ação, conhecimento e status; ii) objetivos compartilhados: conflitos e negociações e iii) divisão horizontal do trabalho.

A simetria na interação pode ser entendida pela proximidade ou distância dos indivíduos em relação ao processo de interação. A respeito da ação, esses estão submetidos a um mesmo rol de atividades; em relação ao conhecimento, apresentam níveis aproximados de clareza sobre o objeto de estudo; quanto ao status, ocupam papeis semelhantes em suas comunidades.

No que se refere aos objetivos compartilhados, Dillenbourg (1999) destaca que os objetivos nem sempre são comuns durante toda a ação do grupo. Em um trabalho colaborativo os objetivos são submetidos a negociações e renegociações constantes, que se tornam mutuamente conscientes e caracterizam os objetivos compartilhados por um espaço de tempo.

Em relação à divisão do trabalho, há que se considerar a distinção entre trabalho cooperativo e trabalho colaborativo. Na cooperação os sujeitos dividem as tarefas em vários fragmentos, resolvem as subtarefas individualmente, de forma assíncrona -resultados são agregados no final para conclusão da atividade. Na colaboração um parceiro executa um nível de tarefa e o outro o meta nível, isso leva a uma divisão "horizontal" do trabalho em camadas de raciocínio. As camadas são altamente entrelaçadas, um assunto monitora e subsidia o outro. 
A divisão horizontal do trabalho é instável na colaboração, os papéis podem mudar a qualquer momento, ao contrário, na cooperação, por ser mais prescritiva, os papéis tendem a permanecer fixos ao longo da execução das subtarefas.

Desse modo, entende-se que estudantes envolvidos em uma atividade colaborativa são autonomamente ativos em seu progresso cognitivo e pelo progresso de seu grupo colaborativo, ligados a um relacionamento corresponsável em relação ao sucesso ou fracasso do grupo. Assim, enquanto os estudantes conduzem suas próprias ações, conferem trocas significativas com o(s) outro(s), tanto no sentido de fornecer informações, soluções e descobertas, quanto no sentido de colaborar com as ideias e críticas.

\section{Winplot e interações matemáticas em contextos ativos e colaborativos de aprendizagem}

Os softwares educacionais se configuram como importantes ferramentas de apoio pedagógico no ensino e aprendizagem da matemática, sendo cada vez mais utilizados por docentes e discentes em métodos ativos, a fim de romper a postura passiva do aluno e motivar para a aprendizagem.

Dito isto, Vieira (2015, p. 3) apresenta a importância do uso dos recursos tecnológicos, destacando a mudança na dinâmica das aulas por meio da utilização do Winplot, como ferramenta de construção de novos conhecimentos e de interação dinâmica entre estudantes, pois "a interação com o software e entre os próprios educandos na realização de uma tarefa ou projeto, incentivou-os a partir de o próprio erro e encontrar o caminho para o acerto, estimulando à colaboração entre os educandos". Desse modo, esses autores alertam que estudantes se tornam condutores do processo de aprendizagem e assumem a tomada da maioria das decisões.

Colaboram nessa reflexão a análise realizada por Santos et al. (2010, p. 48) acerca das possibilidades de uso de softwares matemáticos em sala de aula, pois refletem o desenvolvimento de capacidades intelectuais, a fim de "estimular e contribuir para a busca de mais informações sobre um determinado assunto, promover a colaboração, bem como a interação". Em seus resultados destacam que o conjunto de interações se estabelece como um todo, na tríade professor - aluno - software, para que seja alcançado maior engajamento. 
Diante do exposto, o cenário de construção do conhecimento matemático exige que a interação se estabeleça por meio de uma linguagem própria, que envolve o reconhecimento de proposições, implicações, equivalências, condições de existência, provas, etc. Nesse âmbito em que diferentes pensamentos são formados, pesquisadores se propuseram a estudar o pensamento matemático, entre eles Dreyfus (1991), Gray et al. (1999), Resnick (1987) e Tall (1991).

A respeito do pensamento matemático, Dreyfus (1991) aponta que consiste em uma série de processos que interagem entre si, por meio de representações, visualizações, generalizações, abstrações, entre outros.

Dreyfus sugere que esses processos de interação estão presentes tanto no pensamento matemático elementar quanto no pensamento matemático avançado, distinguindo-se entre eles a complexidade com que são abordados e conduzidos ao longo dos processos, como representação, abstração, classificação, conjecturação, indução, análise e formalização. Por meio da representação e abstração destaca como ocorrem a passagem do pensamento matemático elementar para o avançado.

A representação constitui-se de dois tipos, as simbólicas -que se referem à interação entre signo e significado; e as mentais -que se referem a esquemas internos de comunicação com o mundo exterior. De forma complementar, a visualização -formação e criação de imagens permite intuir e compreender conceitos matemáticos. Tem-se desse modo o estabelecimento de um campo complexo, que envolve todos os processos de interação na construção do conhecimento matemático, entre eles, o campo das representações geométricas. Disso resulta que as representações dos indivíduos estão interligadas, e segundo Dreyfus (1991) podem ser utilizadas de forma simultânea ou alternada, em maior ou menor grau de eficiência.

Nesse campo de interações, colocam-se as representações geométricas, com suas interações próprias de abstração, dedução, visualização etc. Segundo Rautenberg (2013), os sólidos de revolução são obtidos pela rotação de uma área plana em torno de um eixo, chamado eixo de revolução ou rotação. A abstratividade dessa visualização na passagem da representação de 2D para 3D é uma das principais dificuldades de estudantes na construção de conceitos geométricos. Tem-se como exemplo, o caso de Dantas e Mathias (2017) que, ao 
trabalharem com Cálculo Diferencial e Integral, relatam as dificuldades dos estudantes na resolução de problemas com o cálculo de volume de formas de revolução, advindos da tarefa de esboçar ou visualizar uma figura. A esse respeito, Costa (2002) afirma que em pensamento matemático avançado as definições são formuladas e os conceitos formais são construídos por dedução. E nesse sentido, a visualização dos sólidos se relaciona com uma série de processos que interagem entre si, como a visualização e a inferência; na qual as representações mentais ganham existência e auxiliam no processo de desenvolvimento dos estudantes.

Desse modo, ganha forma o pensamento visual-espacial (Costa, 2002), resultante da percepção e da manipulação de imagens construídas pelos sujeitos a partir de interpretações, iterações, abstrações reflexivas, intuições e reconhecimento visual.

Assim, ao trabalhar com a rotação dos sólidos, com a visualização gráfica e com a inferência de objetos geométricos, os discentes interagem com a linguagem matemática avançada e o pensamento visual-espacial. Nesse caso, a representação gráfica possibilitada pelo Winplot pode ampliar a capacidade dos estudantes de representação e expansão da imaginação no campo de conhecimento, ou seja, serve como suporte para aprendizagem de cálculo, pois permite a realização de conjecturas e hipóteses para solucionar situações problemas, possibilitando assim a construção do conhecimento.

\section{Quadro teórico metodológico da pesquisa}

Esta pesquisa toma a perspectiva da investigação qualitativa, que segundo Bogdan e Biklen (1994) tem seus dados recolhidos em contexto naturais, sem a necessidade de levantamento ou comprovação de hipóteses ou de medir variáveis. Portanto, busca apreender as diversas perspectivas dos sujeitos e os fenômenos em sua complexidade, visto que constrói significados estruturados por meio da observação empírica, tomada em função de instâncias concretas de comportamento. Nesse sentido, estruturou-se o planejamento e aplicação de uma oficina voltada a estudantes de cursos de graduação da UNIPAMPA, campus Bagé.

Como instância empírica, consideraram-se as produções configuradas como dados na presente pesquisa e na direção de confirmar tal escolha metodológica, destacam-se as características da investigação qualitativa (Bogdan \& Biklen, 1994), pois a fonte direta de dados é o ambiente natural; a investigação é descritiva; há maior interesse pelo processo do 
que pelos resultados; tende a analisar os dados de forma indutiva e o significado de importância vital em uma abordagem qualitativa de pesquisa.

Nessa direção, destaca-se o ambiente natural, como o lugar subjetivo que os estudantes ocupam no componente curricular de Cálculo II, em função da temática do pensamento matemático em questão; a descrição estendida aos dados gerados durante o processo, entre os quais as resoluções de dois grupos de estudantes e seus posicionamentos; como processo a formatação aplicada, o cuidado do pesquisador direcionado a cada uma das etapas; a parte indutiva da análise argumentativa que se pretende apresentar; além do reconhecimento da importância dos resultados para o entendimento da questão colocada sobre os processos de apropriação pelos estudantes dos estudos acerca de sólidos de revolução.

Os sujeitos de pesquisa são, portanto, definidos em dois grupos de estudantes G1 e G2, que respondem a uma tarefa e a um tutor, também estudante da graduação, matriculado no curso de Engenharia Química e que concluiu Cálculo II no primeiro semestre de 2017. O grupo G1 é composto por duas estudantes do curso de Física-Licenciatura que cursavam Cálculo II no período em que ocorreu a aplicação da oficina e uma terceira integrante, estudante do curso de Engenharia de Energia que cursou Cálculo II há pelo menos dois anos. O G2 é composto por dois estudantes, um estudante do curso de Matemática-Licenciatura e outro do curso de Engenharia de Energia, ambos aprovados em Cálculo II há pelo menos dois anos.

Quanto aos objetivos, recorreu-se à pesquisa exploratória, que para Gil (2008, p. 46) tem a função de "desenvolver, esclarecer e modificar conceitos e ideias, tendo em vista a formulação de problemas mais precisos ou hipóteses pesquisáveis para estudos posteriores". Nessa premissa, objetiva-se levantar elementos da interação, observados durante a aplicação da oficina, revelados na colaboração do ato de trabalhar juntos, em um processo interativo com um objetivo comum.

No âmbito da análise, adota-se a análise argumentativa, que tem como objetivo "documentar a maneira como afirmações são estruturadas dentro de um texto discursivo, e avaliar sua solidez" (Liakopoulos, 2004, p. 219). Entende-se que argumentação é um conjunto de meios sob os quais é possível sustentar pontos de vista, a partir de um encadeamento lógico 
que possibilite ao interlocutor compreender a argumentação, e, portanto, atingir o convencimento sobre uma determinada tese.

A argumentação é assim entendida como uma atividade que congrega um conjunto de afirmações com o objetivo de justificar ou refutar determinada opinião (Liakopoulos, 2004). Desse modo, a análise argumentativa consiste em um trabalho de reconstrução de argumentos, por meio de uma técnica que se inicia com a organização do material, como transcrição das falas e realização de leituras flutuantes, com o objetivo de conhecer em profundidade o material e a perspectiva de análise. Constitui-se então, de um trabalho de busca a respostas a questões norteadoras que serviram de motivação e base para a pesquisa.

Assim, nesta pesquisa, empreende-se a análise argumentativa para investigar elementos de um processo colaborativo de ensino aprendizagem observados durante a aplicação da oficina, com o objetivo de avaliar a solidez das afirmações relatadas e documentar a forma como estas são estruturadas dentro de um texto discursivo. São considerados para fim de análise, os argumentos do tutor, em forma de relato registrado em texto e as observações dos participantes da oficina, em forma de respostas a exercícios e comentários pontuais, anotados durante a resolução dos exercícios propostos na oficina, a fim de perceber elementos da aprendizagem colaborativa e interação na dinamização da construção do conhecimento.

Para a operacionalização da análise, são considerados os cinco passos sugeridos por Liakopoulos (2004): (1) seleção de partes das falas/observações dos participantes que sejam significativas por incorporarem seus pontos de vista; (2) sintetização dos pontos principais das histórias; (3) identificação das partes do argumento; (4) comparação das partes do argumento em um quadro esquemático; (5) apresentação da interpretação em termos de contexto geral. Concluídas estas etapas, cruzam-se as interpretações, a fim de perceber elementos da aprendizagem colaborativa e interação na dinamização da construção do conhecimento, para a interpretação final dos resultados.

\section{Etapas da oficina}

Para a finalidade específica de aplicação das atividades, o objetivo da oficina centrouse em dinamizar o estudo dos sólidos de revolução, por meio da aplicação do Cálculo de Volume, com o propósito de verificar a viabilidade da ferramenta de apoio Winplot para a 
compreensão dos estudantes acerca da visualização espacial em um contexto de aprendizagem colaborativa.

Empreendeu-se, dessa forma, o planejamento da oficina em seis reuniões entre os professores orientadores e o aplicador/tutor da oficina, com duração média de duas horas por encontro. Nesses encontros foram organizados materiais pesquisados, realizadas orientações sobre estudos teóricos acerca da temática da oficina, além de momentos de escolhas de materiais para a sala de aula invertida e de estruturação das atividades que seriam propostas aos estudantes que participassem da oficina.

Nessa direção, primeiramente foi realizada uma pesquisa bibliográfica para analisar os materiais existentes, softwares matemáticos e trabalhos relacionados ao uso de softwares na rotação de sólidos de superfície, a fim de decidir por um mecanismo de ação para a abordagem das atividades envolvendo sólidos de revolução, considerando-se as problemáticas pertinentes a esse estudo. Assim, optou-se pela aplicação da oficina para os discentes que já estudaram ou que se encontravam em estudos relacionados a métodos integrativos e cálculo de volumes de funções rotacionadas, a fim de verificar a relevância e validação da utilização de ferramentas gráficas como complementação de ensino didático.

A oficina foi planejada para uma aplicação em duas horas na sala de Laboratório de Matemática do campus, uma vez que esse espaço na Universidade conta com computadores nos quais estão instalados o programa Winplot. Na oficina, os discentes foram estimulados a trabalhar em grupos formados por escolha própria dos acadêmicos.

O objetivo da oficina é responder questões, previamente estipuladas pelos autores, tanto analiticamente, ou seja, por meio da integração a partir de equações fornecidas, como pelo uso do software Winplot. Além disso, solicitou-se aos participantes que estabelecessem relações entre os objetos de aprendizagem e objetos reais do cotidiano, considerando semelhanças com as formas projetadas pelo software, a fim de permitir inferências e uma maior aplicabilidade e compreensão da temática investigada.

Em relação à sala de aula invertida, foram encaminhados aos inscritos na oficina, um breve tutorial e links de vídeos e apostilas disponíveis na internet sobre o uso do Winplot em atividades matemáticas sobre sólidos de revolução. Essa etapa visou proporcionar aos 
participantes o nivelamento em relação ao contato com o software e a internalização de aspectos relativos ao uso da ferramenta com relativa segurança.

As questões matemáticas propostas na oficina, embora simples, continham diferentes conceitos e aspectos referentes à rotação das funções, de modo que o acadêmico fosse levado a prestar atenção aos detalhes, refletir e deduzir que sólido poderia ser formado ao apresentar a área delimitada por curvas e estabelecer relações com os respectivos volumes, conforme representado na Figura 1.

Figura 1: Equações a serem resolvidas pelos participantes da oficina

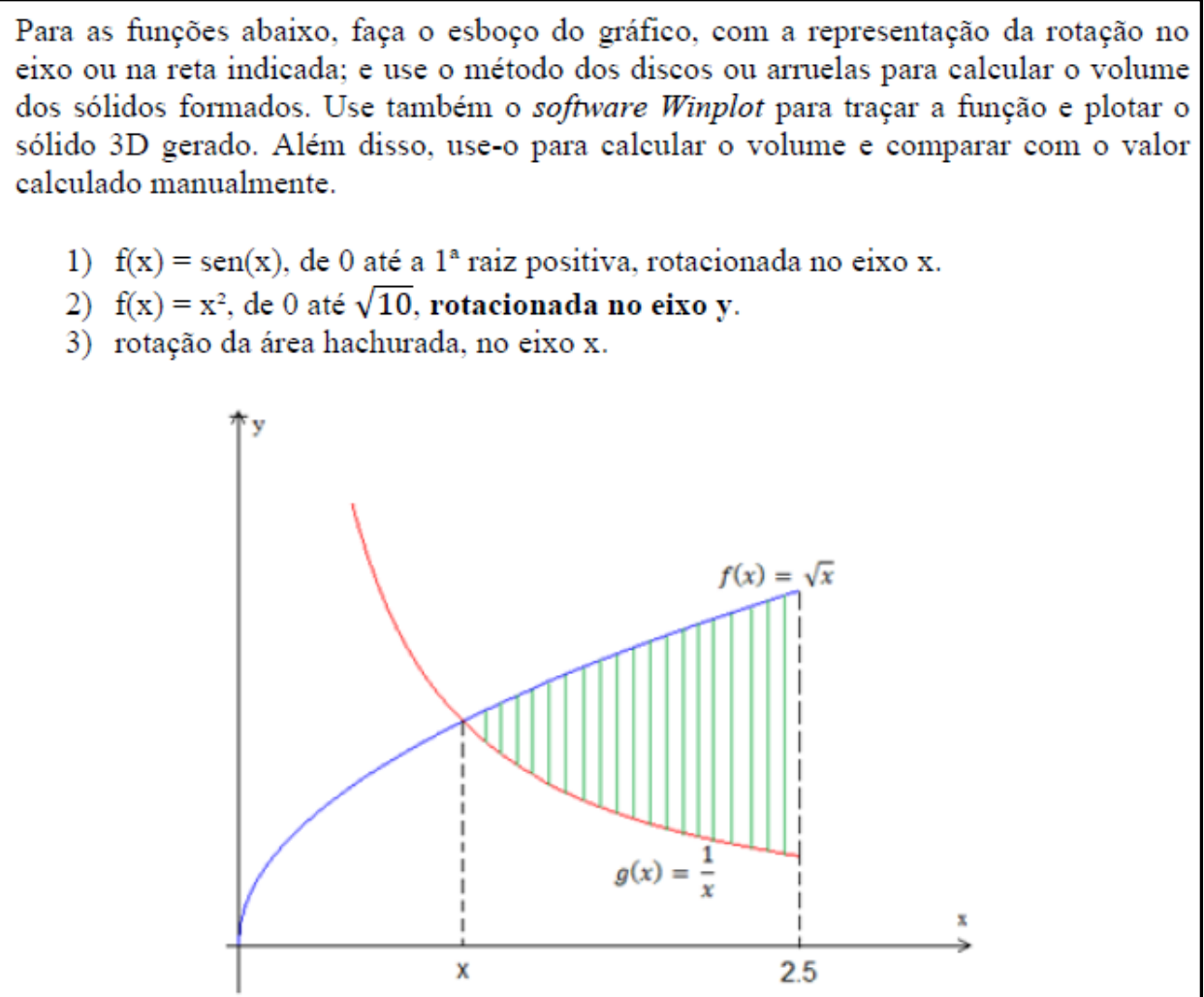

Fonte: Autores (2020)

A Figura 2 apresenta o formulário disponibilizado aos acadêmicos para o cálculo dos volumes na oficina.

Figura 2: Formulário disponibilizado aos participantes

$$
\begin{aligned}
& \text { Rotacionada no eixo x: } V=\pi \int_{a}^{b}[f(x)]^{2} d x \\
& \text { Rotacionada no eixo y: } V=\pi \int_{a}^{b}[f(y)]^{2} d y \\
& \text { Intersecção entre duas funções rotacionadas em x: } V=\pi \int_{a}^{b}\left\{[f(x)]^{2}-[g(x)]^{2}\right\} d x \\
& \text { Identidade Trigonométrica: } \operatorname{sen}^{2}(x)=\frac{1}{2}-\frac{1}{2} \cos (2 x)
\end{aligned}
$$

Fonte: Autores (2020) 
Durante o desenvolvimento dos exercícios propostos na oficina, foram tiradas fotos e recolhidas as folhas utilizadas pelos participantes, para posterior verificação dos elementos de pesquisa propostos no trabalho. Ainda, o tutor se manteve à disposição para colaborar com os grupos e para esclarecer as dúvidas que surgissem, tanto sobre o software como em relação à resolução da atividade matemática. Ao tutor, logo depois da aplicação da oficina, foi solicitada também a elaboração de um "relato" com observações detalhadas acerca de cada etapa do processo. Tal relato constituiu-se importante fonte de dados em análise neste estudo. Na próxima seção, estruturam-se a análise e resultados.

\section{Análise e resultados}

Ao longo do processo de análise foram estruturados os cinco passos sugeridos por Liakopoulos (2004), destacados na seleção observada da atuação e falas dos participantes; na sintetização dos pontos principais dos argumentos observados; na identificação das partes do argumento; na comparação apresentada no Quadro 1; na apresentação da interpretação em termos de contexto geral. Desse processo decorreu a interpretação da interação colaborativa, que é representada no esquema na Figura 3.

Figura 3: Esquema de interação colaborativa

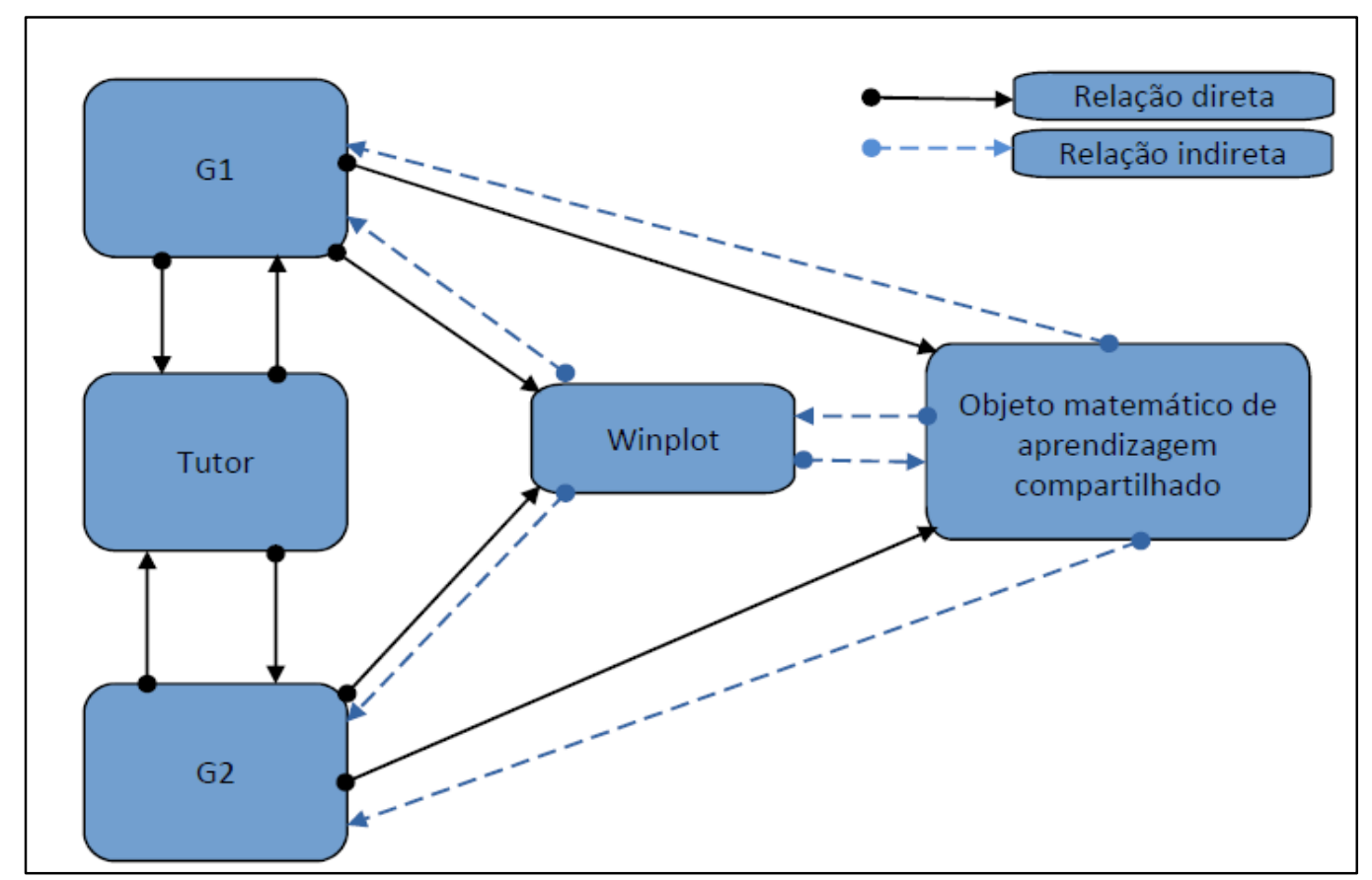

Fonte: Autores (2020) 
Na Figura 3 encontra-se representada a forma da interação colaborativa entre grupos de estudantes; tutor/aplicador da oficina; objeto matemático/objeto de aprendizagem compartilhado e a ferramenta de apoio Winplot. Considera-se que ao longo do processo de análise foram encontrados elementos das três características de aprendizagens colaborativas encontrados em Dillenbourg (1999), simetria, objetivos compartilhados e divisão das tarefas.

No esquema apresentado na Figura 3 são propostas setas de mão dupla, pois a interação colaborativa de aprendizagem é concebida como um processo compartilhado de duplo sentido na busca em alcançar o objetivo compartilhado de aprendizagem, embora seja reconhecido que metas compartilhadas possam ser parcialmente estabelecidas em processos de negociação e renegociação à medida que o trabalho colaborativo se desenvolve.

A relação dos sujeitos com o objeto de aprendizagem está representada na Figura 3 por meio de setas fechadas, dimensionando uma relação direta e horizontal, assim admitida pelo nível de complexidade do problema apresentado, que está de acordo com o nível cognitivo apresentado pelos sujeitos da pesquisa. Considera-se a horizontalidade nessa relação, pois não há distanciamento significativo dos sujeitos em relação ao conhecimento esperado em torno do problema e do objetivo da atividade, que passa a ser compartilhado pelos grupos a partir da apresentação da tarefa pelo tutor. Analogamente, a interação do objeto do conhecimento se estabelece também na interação com a ferramenta Winplot. Esse processo de interação está representado por setas pontilhadas, uma vez que não é direto, ou seja, é intermediado pelo objeto matemático que serve de suporte para a atividade de interação. Destaca-se a interação do objeto matemático com os sujeitos dados igualmente por linhas pontilhadas, pois se estabelece de forma abstrata, não direta, a partir das experiências cognitivas anteriores vivenciadas pelos sujeitos.

$\mathrm{Na}$ sequência são destacados momentos pontuais da oficina e respectivas argumentações decorrentes do processo de análise. Para expor tais argumentos, destacam-se dois âmbitos da argumentação, uma relativa à interação entre os sujeitos e outra, acerca da interação dos sujeitos com os objetos. Explicita-se tal divisão dos âmbitos da análise no Quadro 1 em relação às interações observadas. 
Quadro 1: Interações colaborativas observadas

\begin{tabular}{|l|l|}
\hline Interação entre sujeitos & Interação entre sujeitos e objetos \\
\hline Interação entre G1 e G2 & Interação com material via sala de aula invertida \\
\hline Interação entre os grupos e o tutor & Interação com o objeto matemático em estudo \\
\hline Interação entre o tutor e os grupos & Interação com o software Winplot durante a oficina \\
\hline
\end{tabular}

Destaca-se, porém, que a organização apresentada no Quadro 1 apenas justifica a intenção dos autores de apresentação do contexto geral da análise e de evidenciar os diferentes tipos de interações colaborativas observadas.

\section{Interação entre sujeitos}

Os grupos de estudantes são envolvidos pelo tutor na tarefa de resolver o problema, não há imposição de uma forma de resolução, apenas são disponibilizados os modelos matemáticos e o software Winplot para que recorram, se essa for a opção do grupo, a uma representação dinâmica das etapas da atividade.

A interação entre os grupos e tutor/aplicador da oficina é iniciada imediatamente após a aplicação presencial da atividade. Observou-se liberdade explícita por parte dos dois grupos de estudantes em chamar o tutor em vários momentos durante a tentativa de resolver o problema. Nesse sentido, foram identificados elementos de uma relação horizontal de divisão das tarefas na construção do conhecimento, provavelmente fortalecida pelos elementos de simetria do conhecimento e status entre os pares, uma vez que o tutor/aplicador - mediador da atividade é também um estudante, o que facilita a linguagem e a confrontação imediata das dúvidas recorrentes nos grupos. O contexto das dúvidas é o mesmo atravessado pelo tutor em um tempo não tão distante. Nesse sentido, as experiências dos sujeitos são semelhantes, embora possam ter pontos de vistas distintos quando analisam o mesmo objeto. $\mathrm{O}$ distanciamento entre os membros dos grupos e o tutor é menor devido à simetria do conhecimento, o grau de assimetria seria muito maior em uma relação entre o professor e esses estudantes, pois as experiências ocorreram em tempos e épocas distintas.

Evidencia-se indícios de princípios da aprendizagem colaborativa de Dillenbourg (1999) no grau de divisão do trabalho realizado nos grupos, na simetria da interação entre os sujeitos e no objetivo compartilhado na resolução da tarefa. As interações no grupo mostram relações horizontais que permitem induções, deduções e a construção de uma possível solução 
da questão pelo grupo. Com isso, identificou-se na observação uma leve assimetria acerca do conhecimento do tema por parte dos participantes. Em ambos os grupos, embora tenham avançado por caminhos diferentes, os pares demonstraram o mesmo nível matemático para a resolução da questão.

Negociações nortearam a realização das etapas da tarefa, assim foram evidenciadas atividades de explicação. Por consequência, foram ativados mecanismos cognitivos extras para tomada de decisões nos grupos para responder o problema. De acordo com Dillenbourg (1999), a negociação pode ter um caráter colaborativo mais forte que dar instruções. Aliadas às atividades de explicação, as negociações levam à realização bem-sucedida da tarefa.

Na aprendizagem colaborativa, ao trabalharem juntos, os estudantes também assumem responsabilidades individuais, pois além de interagir e facilitar a aprendizagem dos demais colegas adquirem novas informações, desenvolvendo um diálogo aberto, que promove a autoestima e aprimora hábitos de reflexão e de solidariedade.

O esquema proposto na Figura 3 retrata que não ocorreu interação direta entre os grupos de estudantes G1 e G2, pois, embora essa relação não fosse explicitamente proposta e nem proibida pelo tutor, não foi demonstrada em momento algum da realização das tarefas qualquer atitude que envolvesse os dois grupos na busca pelo resultado do problema. Inferese, que a não interação entre os grupos possa estar relacionada à forte influência do ambiente acadêmico, com suas relações didáticas estabelecidas em cláusulas de um suposto contrato didático $^{4}$, em que os estudantes assumem como inadequada qualquer forma de comunicação com o grupo do qual não participa. O conceito de contrato didático apontado por Dillenbourg (1999) no estudo das aprendizagens colaborativas é encontrado em Brousseau (1986, 1996) e representa uma possibilidade de interpretação para o fato de os dois grupos de estudantes se restringirem à interação aos limites do grupo e da relação com o tutor e objetos de apoio.

\section{Interação entre sujeitos e objetos}

Depois das inscrições confirmadas, foi enviado por e-mail aos participantes o material de apoio para o uso dos recursos do software Winplot durante a oficina. O material era

\footnotetext{
${ }^{4}$ Em Brousseau $(1986,1996)$ o contrato didático diz respeito a um conjunto de regras explícitas em parte, implícitas na maior parte, que determina comportamentos esperados de cada sujeito de uma relação didática.
} 
composto de vídeos, apostilas e tutorial básico para a ferramenta e poderia ser considerado um fator a contribuir para a simetria de conhecimento em relação ao recurso de apoio. No entanto, pode-se inferir que os estudantes dos dois grupos não fizeram uso do material enviado, o que foi demonstrado nas solicitações frequentes ao tutor para ajuda em relação ao uso do Winplot. As dificuldades de uso do aplicativo, representadas em relação a pequenos detalhes como na função do ponto e vírgula no programa, comprovam esse argumento. Contudo, considera-se que devido à fácil acessibilidade da ferramenta, essa dificuldade foi vencida de forma muito rápida pelos grupos, não sendo um fator de interferência no uso da ferramenta ao longo da atividade desenvolvida.

Em contraponto à dificuldade inicial de uso da ferramenta, destaca-se que em continuidade, o software se mostrou essencial e facilitador para as abstrações a partir da visualização dinâmica dos sólidos plotados, além de contribuir para aplicabilidade das integrais definidas no cálculo do volume dos sólidos de revolução.

De forma diversa, o G1 finalizou a atividade recorrendo ao software e o G2 utilizou o recurso durante toda a tarefa. Nesse contexto, foram demonstradas comparações pertinentes que comprovaram os resultados analíticos calculados com lápis e papel com os resultados alcançados com o software. Desse modo, a interação entre o objeto de aprendizagem e o Winplot se mostra intencionada pelos estudantes, que nessa condição, convertem-se em agentes da interação, com o objetivo renegociado e compartilhado no interior de cada grupo de tornar acessível os códigos encontrados no problema, a partir de uma transformação da linguagem para uma nova representação ou linguagem visual. Os acadêmicos ao assumirem essa função conseguem verificar a validade do resultado encontrado e alcançam o objetivo da atividade.

Nesse sentido, a interação entre o objeto compartilhado de aprendizagem e a ferramenta de apoio contribuem significativamente para que os estudantes partam de um pensamento matemático elementar para o pensamento matemático avançado (Dreyfus, 1991). A observação realizada permite admitir que a interação dinamizada pelo software ampliou o âmbito da visualização do problema, do 2D para o 3D, ao possibilitar que novos significados aos objetos fossem alcançados pelos sujeitos agentes da interação. Sobre esse aspecto, Dillenbourg (1999) esclarece que a onipresença das tecnologias nos processos educacionais 
torna cada vez mais difícil estabelecer a fronteira entre a colaboração apoiada ou não pelas tecnologias, contudo, o que se mostra nessa observação é o fato de a tecnologia ter facilitado a visualização e promovido a discussão sobre os elementos observados, o ambiente proporcionou novas perspectivas geradoras de questionamentos no grupo.

O G1 demonstrou expertise para analisar as áreas dos sólidos gerados, foram feitas comparações com as imagens plotadas (macarrão conchinha, casca de sorvete). A acadêmica que já havia cursado Cálculo II há mais de dois anos demonstrou maior segurança e domínio nas operações básicas de matemática para resolver as integrais, no entanto, todas as integrantes do grupo demonstraram conhecimentos próximos para analisar as áreas e para decidir quais equações usar na resolução da tarefa.

Essa aproximação em torno dos conhecimentos é caracterizada em Dillenbourg (1999) como simetria de conhecimento, embora uma leve assimetria seja considerada igualmente promissora em processos colaborativos, pois, para esse autor, geralmente levam a ocorrência de interações conflitantes, sendo essas bem-vindas quando se trata de aprendizagem colaborativa.

O G1 conseguiu desenvolver os cálculos e gerou os sólidos no Winplot. Foi demonstrada, por esse grupo, uma discussão focada para o entendimento dos processos de cada problema. Durante a execução da atividade foram cometidos erros simples, por desatenção ou talvez por ainda não terem alcançado um conhecimento significativo em relação à aplicação dos limites da integração. No entanto, durante todo o processo foi externada forte articulação do trabalho entre os pares, afinal, enquanto uma tarefa era executada individualmente, os demais prestavam atenção à cada etapa da atividade em andamento. Nesse sentido, infere-se a ocorrência da divisão horizontal do trabalho no sentido apresentado por Dillenbourg (1999), pois esse grupo desde o início da atividade recorreu ao software, e quando uma das integrantes plotava funções no Winplot, as demais participavam fazendo conjecturas e dando opinião sobre os cálculos.

Desse modo, o G1 conseguiu plotar as funções e gerar os sólidos, porém as integrantes manifestaram dificuldades em verificar o valor do volume pelo software e para isso recorreram ao tutor, que mediou o processo e auxiliou na validação de suas respostas. Essa relação com o tutor denota o que Dillenbourg (1999) caracteriza como simetria de status, pois 
observa-se que os agentes envolvidos nessa ação apresentam um status semelhante em relação à comunidade da qual fazem parte, todos são acadêmicos na mesma instituição de ensino. Cabe destacar que essa condição coloca os estudantes em um processo em que as relações tendem a não ser intimidadas por hierarquias, favorecendo significativamente a interação.

Ainda, das observações realizadas acerca do G1, constatou-se que o foco esteve mais voltado para as interações possíveis no ambiente gráfico. Nesse grupo, as integrantes buscaram entender mais o processo de rotação das funções e visualização 2D, assim, foram estabelecidas comparações a partir do traçado no papel, para o 3D, obtido com o programa. A Figura 4 representa a resolução no software de uma das atividades desenvolvidas na oficina pelo grupo G1.

Figura 4: Representação do problema três

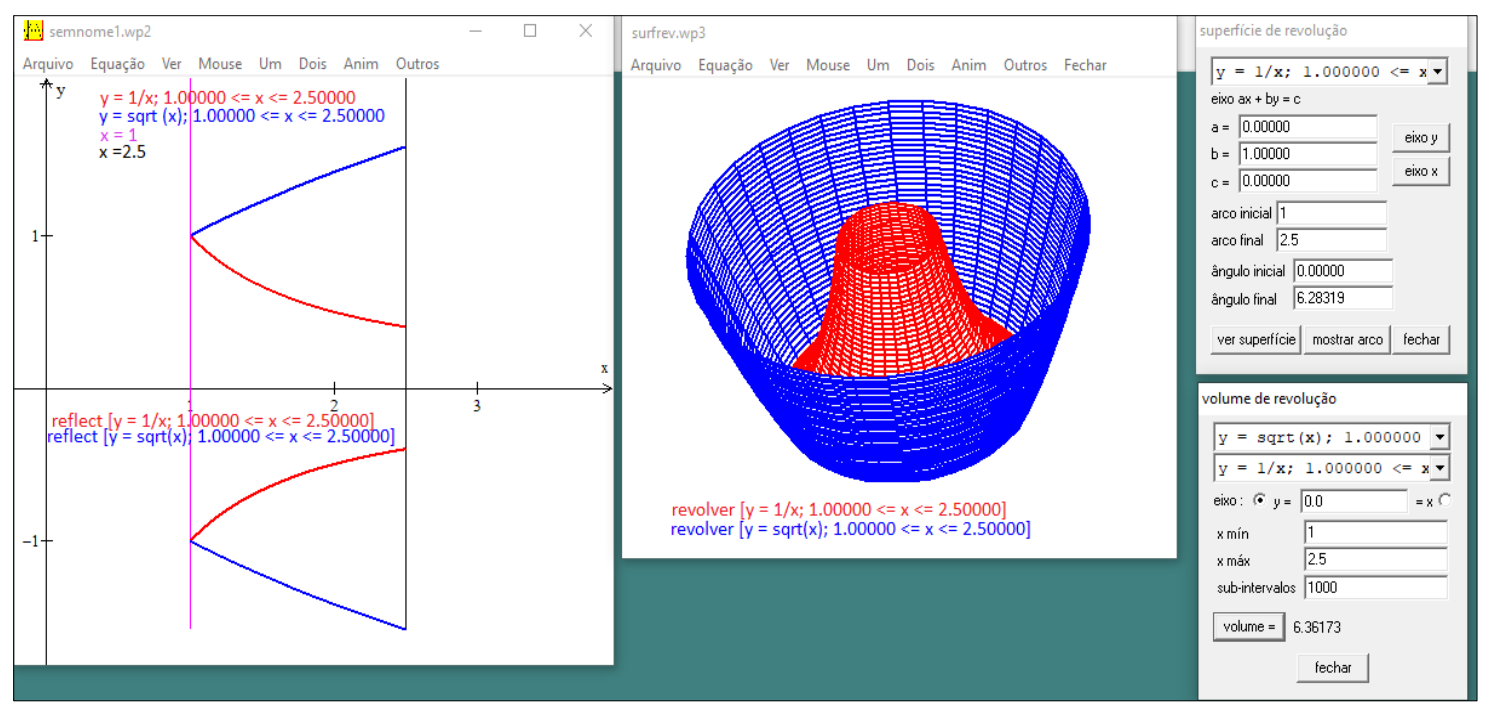

Fonte: Autores (2020)

Sobre a atuação do G2, observou-se um conhecimento aprofundado dos dois estudantes em Álgebra e Cálculo. Ao contrário do que apresentou G1, no início da atividade os integrantes do G2 estavam focados mais nos cálculos analíticos que no desenvolvimento pelo software, de maneira que, foram expressadas dificuldades iniciais em usar o Winplot.

Os membros do G2 recorreram às conjecturas dos sólidos antes de lançar mão de plotagens. Usaram de diferentes caminhos para calcular o volume como: integrações duplas e triplas em um processo investigativo, comparando os sólidos e o resultado com integrais duplas, recorrendo também a limites de integração. Somente depois de conclusões matemáticas encaminhadas é que recorreram ao software para girar a função encontrada nos 
eixos, a fim de identificar a diferença dos sólidos gerados. A decisão tomada pelo grupo indica que a interação com o software confirmou as suposições matemáticas elaboradas pelo grupo. As constantes explicações e negociações em torno da resolução da tarefa podem ser observadas na imagem destacada na Figura 5.

Figura 5: G2 debate sobre hipóteses levantadas

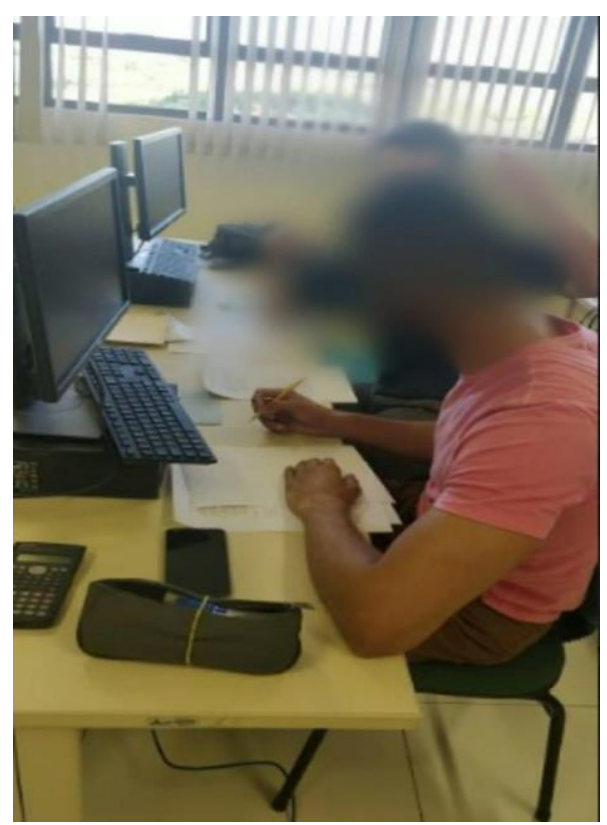

Fonte: Autores (2020)

Nesse sentido, atividades de explicação, desacordos e regulação mútua são apontadas por Dillenbourg (1999) como atividades que contribuem para gerar mecanismos extras para obtenção de conhecimentos. A interação propiciada pela tarefa e os recursos disponibilizados contribuiu fortemente para as atividades e mecanismos extras de aprendizagens observados.

Os integrantes do G2 demonstraram percepções iniciais diferentes para a resolução, porém a aprendizagem colaborativa, conforme Dillenbourg (1999), não é um mecanismo que exclui a aprendizagem individual. Aprender em colaboração significa também aprender sozinho.

Nesse sentido, a observação realizada reiterou momentos em que os estudantes realizaram leitura do problema, sistematizaram uma construção individual para resolver a questão e apresentaram mecanismos de aprendizagem de dedução. No entanto, a partir do momento que exibem entre si suas ideias e interpretações, as atividades de conversa e explicação sobre a solução obtida promovem também a ajuda mútua e a tomada de decisão 
sobre um caminho de solução do problema, revelam indícios de mecanismos de aprendizagem colaborativa.

Diante do exposto, o G2 foi além do objetivo de resolver o problema proposto na oficina, pois os membros desse grupo também realizaram pesquisa de informações em portais eletrônicos sobre os cálculos efetivados e poucas vezes pediram auxílio ao tutor para questões matemáticas. Características como autonomia e independência foram pronunciadas quando os componentes do grupo optaram por uma divisão horizontal do trabalho (Dillenbourg, 1999) em camadas entrelaçadas de raciocínio. Não houve divisão vertical, pois mesmo quando, a princípio, se dedicaram individualmente a alguma subtarefa, a relação de interdependência entre as tarefas foi mantida.

Para concluir esta análise, destaca-se que para Dillenbourg (1999) uma situação é considerada colaborativa se atende aos seguintes critérios: simetria na interação, objetivos comuns e divisão do trabalho entre os pares da colaboração. Diante disso, na interação dos grupos G1 e G2 com o Winplot, situação mediada pelo objeto matemático compartilhado, evidenciou-se a leveza dos sujeitos na construção dos gráficos e na solução dos problemas propostos na oficina. O software permitiu com certo grau de simplicidade que em minutos fosse realizada a representação gráfica solicitada no problema proposto, o que possibilitou também a exploração de conceitos como: rotação dos eixos e o cálculo dos volumes pedidos.

Desse modo, o grau de simetria de ação, de conhecimento e de status, evidenciados por Dillenbourg (1999), tornam-se novamente explícitos, à medida em que o mesmo rol de atividades foi proposto a todos, a habilidade dos integrantes na resolução da tarefa foi próxima e o pertencimento a uma mesma comunidade acadêmica favoreceu a comunicação dos integrantes dos grupos entre si, tornando-se esses aspectos pontos fortes para facilitar a obtenção dos resultados atingidos com sucesso na resolução do problema.

Destaca-se ainda o diálogo aberto entre os participantes e a divisão horizontal das tarefas, pois não houve imposições hierárquicas ou verticais, todos os membros trabalharam em todas as etapas da construção da atividade com o Winplot, colaborando mutuamente ao longo da realização de cada etapa da atividade. A visualização gráfica proporcionada pelo Winplot igualmente contribuiu para as discussões e decisões tomadas em torno objeto de estudo e do objetivo compartilhado. Por fim, afirma-se em consonância com a perspectiva de 
aprendizagem colaborativa de Dillenbourg (1999) que os pares demonstraram nos seus grupos, mais ou menos o mesmo nível de conhecimento, realizaram ações articuladas e trabalharam juntos para atingir o objetivo comum de realizar a tarefa proposta na oficina.

\section{Considerações finais}

O estudante quando submetido a um método que valoriza aprendizagens colaborativas, do mesmo modo que é corresponsável por sua aprendizagem, torna-se colaborador da aprendizagem de seus colegas, o que amplia o desenvolvimento de sua autonomia. Durante o desenvolvimento da oficina, a interação entre os componentes dos grupos G1 e G2 mostrou o engajamento de agentes responsáveis pelo próprio aprendizado. O tutor cumpriu a finalidade de mediar o processo, participando das decisões horizontais dos colegas para cumprir as tarefas, tomando na maior parte das vezes também a postura de colaborador.

A aprendizagem colaborativa ocorreu de forma contínua, negociada e natural, desde as primeiras hipóteses levantadas, nas definições das estratégias, na formalização dos procedimentos e conhecimentos matemáticos para a resolução dos problemas. Importante destacar que em certos momentos aconteceu a comparação com objetos do dia a dia, essa associação geralmente não acontece em aulas apenas teóricas.

Concluindo, o software como ferramenta de apoio pedagógico, validou a visualização e comparação dos sólidos gerados com objetos reais, foi, portanto, um recurso didático significativo para a interação dos estudantes com o objeto de aprendizagem Cálculo. Na comparação das figuras geradas com objetos conhecidos, recorreram à formulação de conjecturas, um mecanismo de aprendizagem importante para a formação de conceitos e para o desenvolvimento do pensamento matemático avançado.

\section{Referências}

Bogdan, R. \& Biklen, S. (1994). Investigação qualitativa em educação: Uma introdução à teoria e aos métodos. Porto Editora.

Bonwell, C. C. \& Eison, J. A. (1991). Active learning: Creating excitement in the classroom. DC School of Education and Human Development, George Washington University.

Brousseau, G. (1986). Fondements et méthodes de la didactique des mathématiques. Recherches en Didactique des Mathématiques,7(2), 16-33. $\quad$ https://revuerdm.com/1986/fondements-et-methodes-de-la/ 
Brousseau, G. (1996). Os diferentes papéis do professor. Em C. Parra e I. Saiz (Eds.), Didática da Matemática: Reflexões psicopedagógicas (pp. 54-78). Artes Médicas.

Costa, C. (2002). Processos mentais associados ao pensamento matemático avançado: Visualização. Anais do Encontro da seção de Educação Matemática da Sociedade Portuguesa de Ciências da educação, Coimbra, Portugal.

Dantas, S. C. \& Mathias, C. V. (2017). Formas de Revolução e Cálculo de Volume. Ciência e Natura, 1(39), 142-155. https://www.redalyc.org/pdf/4675/467549116016.pdf.

Dillenbourg, P. (1999). What do you mean by collaborative learning? In P. Dillenbourg (Ed.), Collaborative-learning: Cognitive and computational approaches (pp. 1-19). Elsevier.

Dreyfus, T. (1991). Advanced mathematical thinking processes. In D. Tall (Ed.), Advanced mathematical thinking (pp. 25-41). Kluwer.

Felder, R. M. \& Brent, R. (2009). Active Learning: An introduction. ASQ Righer Education Brief, 2(4),

$1-5$. https://www.researchgate.net/publication/242102584_Active_learning_An_introduction.

Gil, A. C. (2008). Métodos e Técnicas de Pesquisa Social (6.a edição). Atlas.

Gray, E., Pinto, M., Pitta, D. \& Tall, D. (1999). Knowledge construction and diverging thinking in elementary and advanced mathematics. Educational Studies in Mathematics, (38),

111-133. https://idp.springer.com/authorize/casa?redirect_uri=https://link.springer.com/article/10.10 23/A:1003640204118\&casa token=XV2iz187L2gAAAAA:V-QwcUiSeKzFvmP6vUMrW5thzM-cabtaZeQzyHQYiGRFKomJia6s3tB_BZ8XQmLatotYw7yPhcJ3x_LoX4.

Liakopoulos, M. (2004). Análise argumentativa. Em M. W. Bauer e G. Gaskell (Eds.), Pesquisa qualitativa com texto, imagem e som: Um manual prático. Vozes.

Moraes, S. P. G. (2008). Avaliação do processo de ensino e aprendizagem em Matemática: Contribuições da teoria histórico-cultural [tese de doutorado, Universidade de São Paulo]. Repositório da USP. https://www.teses.usp.br/teses/disponiveis/48/48134/tde-16032009145709/publico/Silvia_Pereira_Gonzaga_de_Moraes.pdf.

Pacheco, J. A. D. \& Barros, J. V. (2013). O Uso de Softwares Educativos no Ensino da Matemática. Diálogos: Revista de Estudos Culturais e da Contemporaneidade, (8), 5-13. http://www.revistadialogos.com.br/Dialogos_8/Adson_Janaina.pdf.

Rautenberg, R. R. (2013). Os teoremas de Pappus para os sólidos de revolução [dissertação de mestrado, Universidade Tecnológica Federal do Paraná]. Repositório Institucional UTFP. http://repositorio.utfpr.edu.br/jspui/bitstream/1/471/1/CT_PROFMAT_M_Rautenberg,\%2 0Robson\%20Raulino_2013.pdf.

Resnick, L. (1987). Education and learning to think. National Academy Press.

Sadovsky, P. (2007). O Ensino de Matemática Hoje: Enfoques, Sentidos e Desafios. Editora Ática.

Santos, R., Loreto, A. B. \& Gonçalves, J. L. (2010). Avaliação de softwares matemáticos quanto a sua funcionalidade e tipo de licença para uso em sala de aula. Revista de ensino 


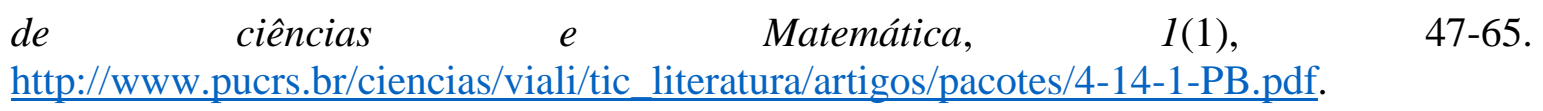

Tall, D. (1991). The psychology of advanced mathematical thinking. In D. Tall (Ed.), Advanced mathematical thinking (pp. 3-21). Kluwer.

Torres, P. L. \& Irala, E. A. F. (2014). Aprendizagem colaborativa: Teoria e prática. Em P. L. Torres (Ed.), Complexidade: Redes e conexões na produção do conhecimento (pp. 61-93). SENARPR.

Vickery, A. (2016). Aprendizagem ativa nos anos iniciais do Ensino Fundamental (H. O. Guerra, Trad.). Penso Editora Ltda.

Vieira, G. (2015). O uso do software Winplot na aprendizagem matemática em sala de aula: Uma inovação pedagógica? [dissertação de mestrado, Universidade da Madeira]. Repositório Institucional da Universidade de Madeira. http://hdl.handle.net/10400.13/1162.

Weltman, D. (2007). A comparision of traditional and active learning methods: An empirical investigation utilizing a linear mixed model [tese de doutorado, University of Texas at Arlington]. $\quad$ https://rc.library.uta.edu/uta-ir/bitstream/handle/10106/734/umi-uta1921.pdf? sequence $=1$ \&isAllowed $=\mathrm{y}$.

Autores

Sonia Maria da Silva Junqueira

Doutora em Educação Matemática pela Pontifícia Universidade Católica de São Paulo (Brasil). Professora Adjunta III da Universidade Federal do Pampa (UNIPAMPA, Brasil) e docente permanente no Programa de Pós-Graduação Mestrado Acadêmico em Ensino (MAE).

Temas de pesquisa: Aprendizagens ativas, colaborativas, solidárias e críticas, em todos os níveis educativos. E-mail: soniajunqueira@unipampa.edu.br

Leandro Blass

Doutor em Modelagem Computacional pela Universidade do Estado do Rio de Janeiro (Brasil). Professor adjunto A da Universidade Federal do Pampa (UNIPAMPA, Brasil). Temas de pesquisa: Modelagem dos fenômenos de transporte, com ênfase em Modelagem de deposição de parafinas em dutos de petróleo; Modelagem e simulação dos fenômenos de transferência de calor em pavimentos flexíveis; Aprendizagens ativas no Ensino Superior; Avaliação do desempenho discente; Rubricas avaliativas. E-mail: leandroblass@unipampa.edu.br

Lucas Capello

Graduando no curso de Engenharia Química pela Universidade Federal do Pampa (UNIPAMPA, Brasil). Membro do Projeto de Pesquisa Laboratório de Investigações Matemáticas Ativas. Temas de pesquisa: Metodologias ativas; Aprendizagens colaborativas. E-mail: lucascapello@hotmail.com 